\title{
Toward a Common Ground for Gravity and Optics
}

\author{
Jose L. Parra \\ Department of Physics, Florida International University, Miami, FL, USA \\ Email: JLparra@fiu.edu
}

How to cite this paper: Parra, J.L. (2018) Toward a Common Ground for Gravity and Optics. Journal of Applied Mathematics and Physics, 6, 1896-1906. https://doi.org/10.4236/jamp.2018.69161

Received: July 20, 2018

Accepted: September 22, 2018

Published: September 25, 2018

Copyright (C) 2018 by author and Scientific Research Publishing Inc. This work is licensed under the Creative Commons Attribution International License (CC BY 4.0).

http://creativecommons.org/licenses/by/4.0/

\begin{abstract}
A long enough period of observation of the Sun's gravitational dragging effects by using a modified Cavendish's balance output of experimental evidence shows new patterns. Those patterns can be explained assuming that the Sun has a torus with rotation, precession, and nutation. This purpose of this paper is to introduce the frequencies of all those movements. The torus's rotational period can be used to explain the Sun's magnetic pole reversal. Utilizing a modified Cavendish's balance showed an output of dragging forces stronger than the attraction between the gravitational masses. This tool afforded this research a new experimental possibility to a more precise determination of the Universal Gravitational Constant Big G. Moreover, the dragging forces directly affect any volume of mass, which includes the atmosphere. This paper shows a correlation between the Sun's dragging peaks and density of the air squared. The aforementioned correlation and the inverse cubic relation with the distance to the Sun are common for the dragging and tide forces providing the possibility that tidal forces are also a gravitational dragging consequence. The last 2017 total Solar eclipse created a new temporal reaction on the modified Cavendish's balance. That temporal pattern looks as the spatial pattern created by an opaque disk. This similarity allows the researcher to calculate that the dragging forces are transmitted by photons with spatial periodicity of value $\lambda=6.1 \mathrm{~km}$.
\end{abstract}

\section{Keywords}

Spinning Black Holes, Kerr's Metric, General Relativity, Torsion

Measurements, Gravitational Constant G, Rotation, Precession, Solar Cycles

\section{Introduction}

In 2015, Schlamminger [1] reported a synthesis of the best experimental results 
on the determination of the oldest constant in physics. That constant was a mathematical consequence of the Newtonian theory of gravity, which is known as the Universal Gravitational Constant or Big G. Some inconsistency is visible in [1] because all experimental results, besides having very little standard deviation, do not show overlapping values. Due to the lack of clear evidence indicating the best result, the scientific community recommended the use of the average of all best outcomes. The last two years have been used to accumulate experimental data without a new final report about the value of Big G. This is notable as there is limited research on this scientific concern in the present literature. Only some theoretical work as the produced by Stirling [2] shows a meticulous revision of the practical equation about gravitational interactions.

In 2016, the author of the present study observed an apparent inverse relationship between the experimental values of Big G and the distance to the Sun, after making a three-dimensional graph with the Big G's values versus time. A possible correlation between data and the available theory was published by the Author in 2017 [3]. This was a desperate publication to indicate that measures of Big G should not be average. More effectively, the data should be plotted on a graph; and from there the observation of the output pattern could help to eliminate the systematic variation of the experimental values associated with the influence of the Sun.

The author has accumulated 612 experimental points, one point per day, observing the dragging effect of the Sun on the angular position of his gravitational balance. The pattern visible within the figures introduced here indicated the presence of at least four cycles. The author claims that the $24^{\text {th }}$ Solar Cycle was completed on November $21^{\text {st }}, 2017$ and the $25^{\text {th }}$ Solar Cycle commenced on July $21^{\text {st }}, 2018$. These dates may cause some controversy among scholars because that is not what is seen on the surface of the Sun. This discrepancy could be understood by considering that the author is reporting what he suggests is happening in the nucleus of the Sun and not on the surface of the Sun.

Additionally, it could be useful to mention the dragging force, which produces precession forces over bodies with extension, can also effect the mass-points movements. Those dragging forces experimentally look as Coriolis's forces but with an important distinction, the dragging forces are real.

\section{One Year Data}

The Author in [3] included only six months of observation. The minimal quantity of data made the author fail at naming the observed frequencies. The Author incorrectly mentioned a nutation within a six-day period and a precession over a 20-day period. Currently, the Author can claim, by the correlation between the dragging perturbations and the Sun's spots numbers, that the Sun's torus has four global movements. They are:

1) Rotation with a periodicity of 22 years.

2) Precession with a periodicity of two years. 
3) Long-period nutation of 20 days, and

4) Short-period nutation of six days.

Figure 1 shows last three periodicities graphically. The latest point, which reflects the 22 years rotation, has a different color in Figure 2 for clarity.

The points which apparently connected with the $25^{\text {th }}$ Solar cycle, the first of which was discovered by Schwabe [4], were introduced in Figure 2. It is important to note this figure because it indicated that the Sun's magnetic field flipped as is presently accepted. The sun's torus flips every 11 years. Subsequently, if the north was evident for 11 years the next flip would result in 11 years of observing the south magnetic pole.

Five points were introduced to show the passing of time. The five points are as follows: a) January $1^{\text {st }}, 2017$; b) July $1^{\text {st }}, 2017$; c) the end of the 24 SC and the beginning of the transition 24th-25th SC, November $21^{\text {st }}$, 2017; d) the beginning of the 25th SC, July $28^{\text {st }}, 2018$; and e) the date of the first sunspot with the magnetic field reverse.

The team developer of the web page Spaceweather.com [5] published on August $26^{\text {th }}, 2018$ "New sunspot AR2720... appears to be the first big sunspot of the next solar cycle, Solar Cycle25". In this way, two independent observations are made: 1) what is happening inside the Sun, and 2) the other counting sunspots on the surface of the Sun, which completely match their conclusion. The signal from the Sun follows the same pattern for more than a year and a half; then suddenly, that pattern is completely flipped. As a result, a month later the magnetic field of a sunspot, on the surface of the Sun, appears flipped. Maybe this experimental evidence is not conclusive yet, but it provides important support to the model introduced by the author.

The author [3] with only six months of observation, reported that Big G could have the value of $(6.6114 \pm 0.0031)^{*} 10^{-11} \mathrm{Nm}^{2} \cdot \mathrm{kg}^{-2}$ and a dragging coefficient of $(0.0616 \pm 0.0031)^{\star} 10^{-11} \mathrm{Nm}^{2} \cdot \mathrm{kg}^{-2}$. However, with 612 points, it is possible to make a more reliable estimation of the unknown gravitational parameters. The Kerr's dragging frequency Equation (1) has two directional components. Each

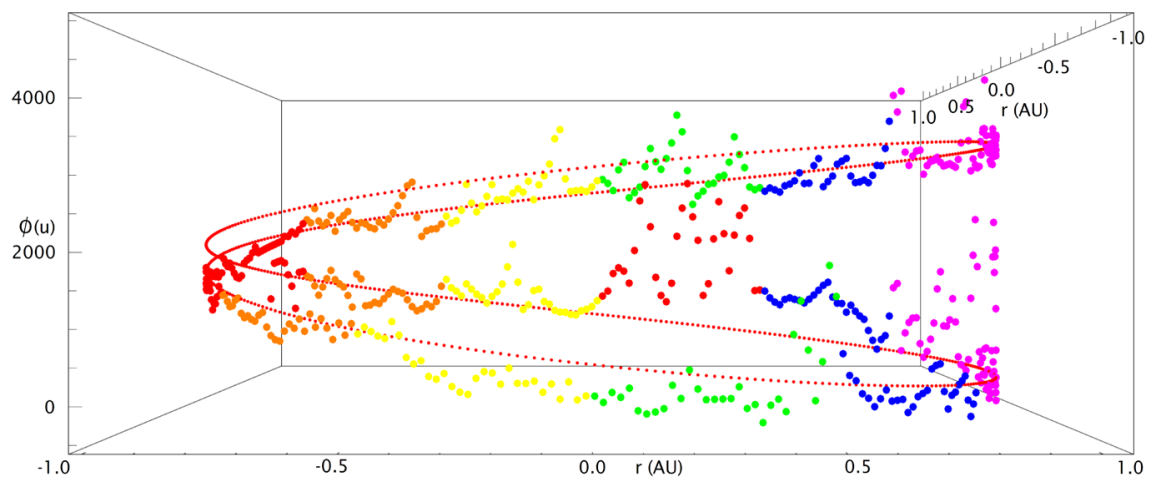

Figure 1. Every day of a month with same color, January and December (on the right) sharing same color as June and July (on the left). The little red points come from a function with two years of a period. The March-2018 points are in red to distingue them from the points in green of March-2017. 


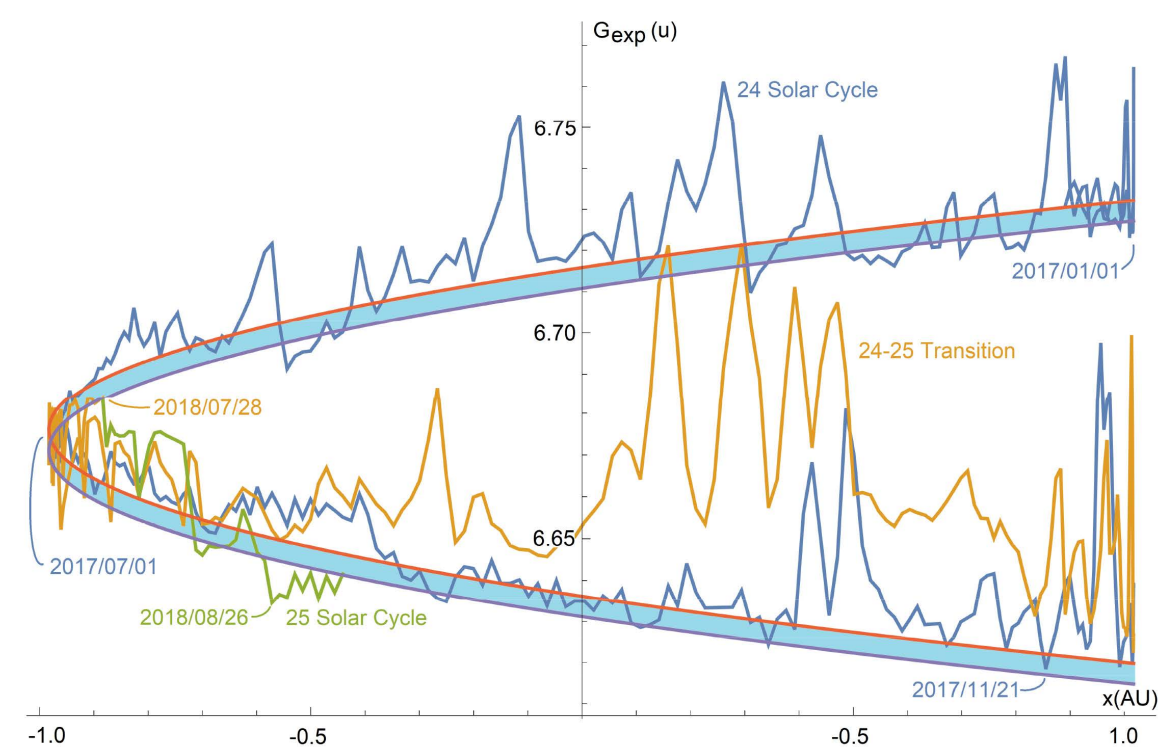

Figure 2. Big $\mathrm{G}$ values during 612 observations. The ribbon covers the points not affected by nutation. The $24^{\text {th }}$ Solar Cycle (SC) in blue. The transition $24^{\text {th }}-25^{\text {th }}$ SC in Orange. The $25^{\text {th }} \mathrm{SC}$ in green.

one produces a bigger effect depending on three factors: the latitude where the experiment is carry out, the hours of the measure, and the direction of the axis of rotation of the Cavendish's balance.

$$
\overrightarrow{\boldsymbol{\Omega}}_{p}=\frac{G}{c^{2} \rho^{5}}\left[3(\overrightarrow{\boldsymbol{J}} \cdot \overrightarrow{\boldsymbol{\rho}}) \overrightarrow{\boldsymbol{\rho}}-\rho^{2} \overrightarrow{\boldsymbol{J}}\right]
$$

where $\Omega$ is the precession frequency of anybody with extension, $G$ is the Universal Gravitational Constant, $c$ is the speed of light, $\rho$ is the radial distance between the source and the spinning body, and $J$ is the angular momentum of the source; in our case the Sun's nucleus torus.

On average, in Miami, the radial component plays the most important role according to the experimental data. Equation (1) was reduced to include only the projection of the precession angular frequency in the direction of the apparatus axis of rotation. By separating constants from variables,

$$
\frac{a^{3} c^{2} \Omega_{p}}{3 J} \approx G\left(\frac{a}{\rho}\right)^{3} \cos (\theta) \cos (\alpha)
$$

where a's value is one Astronomical Unit (1AU), and $\alpha$ is the angle between the Sun-Earth direction and the direction of apparatus axis of rotation. At this time, it is important to mention that even in the case that $\alpha$ is nine degrees, the apparatus should show some microscopic precession because that is mechanically possible working with strings attached close to the balanced center of gravity.

Equation (2) showed that, upon first approximation, a lineal dependence could be assumed between the experimental dragging effect and the inverse of third power of the distance to the source and the cosine of the torus' direction. This connection could be made by using Equation (3), 


$$
G_{\text {exp }}=G_{N E}+G_{(a, \alpha)}\left(\frac{a}{r}\right)^{3} \cos \left(\theta_{\text {torus }}\right)
$$

where $G_{\text {exp }}$ is the experimental observation, $G_{N E}$ is the Universal Gravitational Constantor Big G used by Newton and Einstein, $G_{(a, a)}$ is the perturbative effect coming from the rotation of the nucleus of the Sun, $a$ and $r$ are the average and instantaneous Sun-Earth separation, and $\theta_{\text {torus }}$ is the angle between the torus axis of rotation and the line defined by the centers of the Sun and the Earth. This angle has an average value of 45 degrees, from the Earth's point of view; however, its daily value can go from 45 to 135 degrees. That fluctuation is created by the superposition of the nucleus rotation, the precession of the nucleus, and both nutation's. Equation (3), having a constant plus a no sinusoidal temporal variable, correlated well with the opinion of Pitkin [6] and Desai [7].

The equipment used in this experiment guarantees a lineal relationship between the angle of deflection $\Phi$ and the experimental observation of Big G. The numerical connection $G_{D e c}=6.676 \times 10^{-11} \mathrm{Nm}^{2} \cdot \mathrm{kg}^{-2}$ and $G_{J u l}=6.671 \times 10^{-11}$ $\mathrm{Nm}^{2} \cdot \mathrm{kg}^{-2}$ allows the creation of Figure 2. It is important to note there that in the author's apparatus does not eliminate the angular $\theta_{\text {torus }}$ influence on the results because the attractive non-oscillating masses are fixed in space in contraposition to the Cavendish's method that alternates the position of these masses in respect to the oscillating ones. A ribbon-like visual covers the points with higher probability to correlate $G_{\text {exp }}$ with the date of the experiment in Equation (3). It was super-imposed in Figure 2 by excluding the points affected by strong nutation. The width of the ribbon can be used as an error-criterion. The two gravitational factors become $G_{N E}=(6.6735 \pm 0.0025)^{\star} 10^{-11} \mathrm{Nm}^{2} \cdot \mathrm{kg}^{-2}$ and $G_{(a, \alpha)}=(0.0753 \pm$ $0.0002)^{\star} 10^{-11} \mathrm{Nm}^{2} \cdot \mathrm{kg}^{-2}$.

The intention of the experiment introduced in this paper is to mathematically characterize the pattern made by the angle of a torsion balance with time. The existence of that pattern suggests the possibility of using this technique to improve the precision of Big G. This objective can be achieved by collecting data over extended period's time by utilizing 24-hour periods and seeking data in 15-minute increments. The author does not have the financial capacity or resources to pursue this project.

\section{Weather in Miami}

The author wants to call attention to a permanent correlation between the picks of the long-period nutation solar cycle and the square of the air density. A theoretical model will not be introduced in this paper because only one station cannot bring enough statistical confidence. To produce some confidence on the parameter correlates, the author used the Sun's perturbations from its apparatus (the only information available to him) and the pressure and temperature of an outside point that can be checked if desired. The reference point was Miami International Airport and MesoWest [8] the software used to get data in real time. The air density was calculated using the ideal gas equation. Figures 3(a)-(c) 


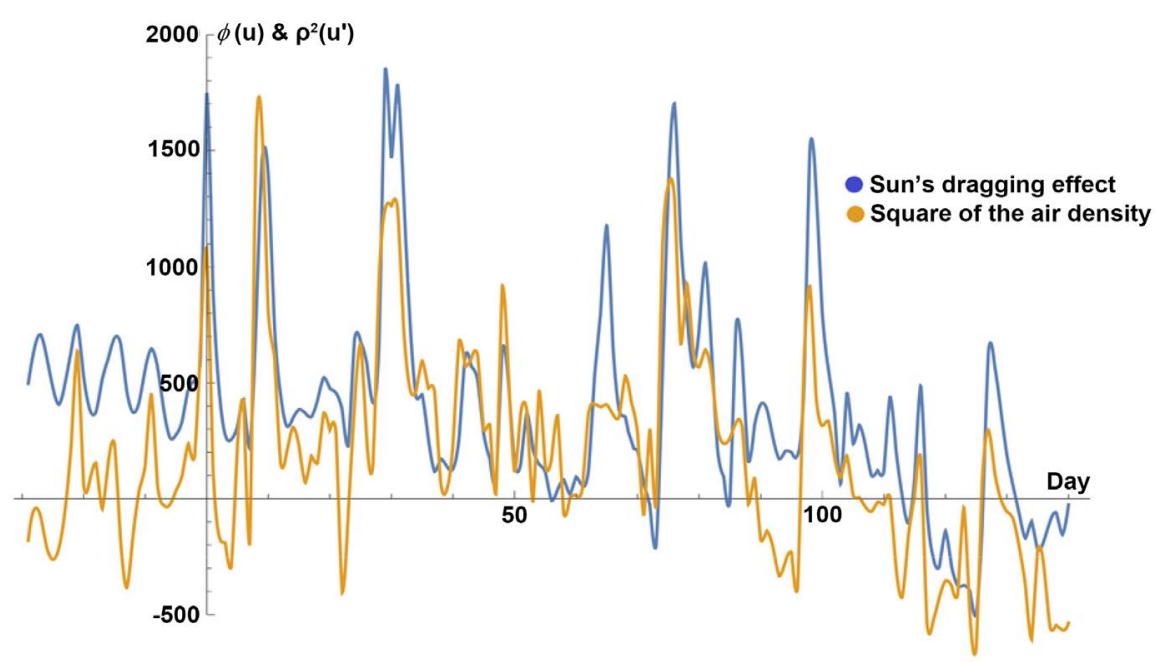

(a)

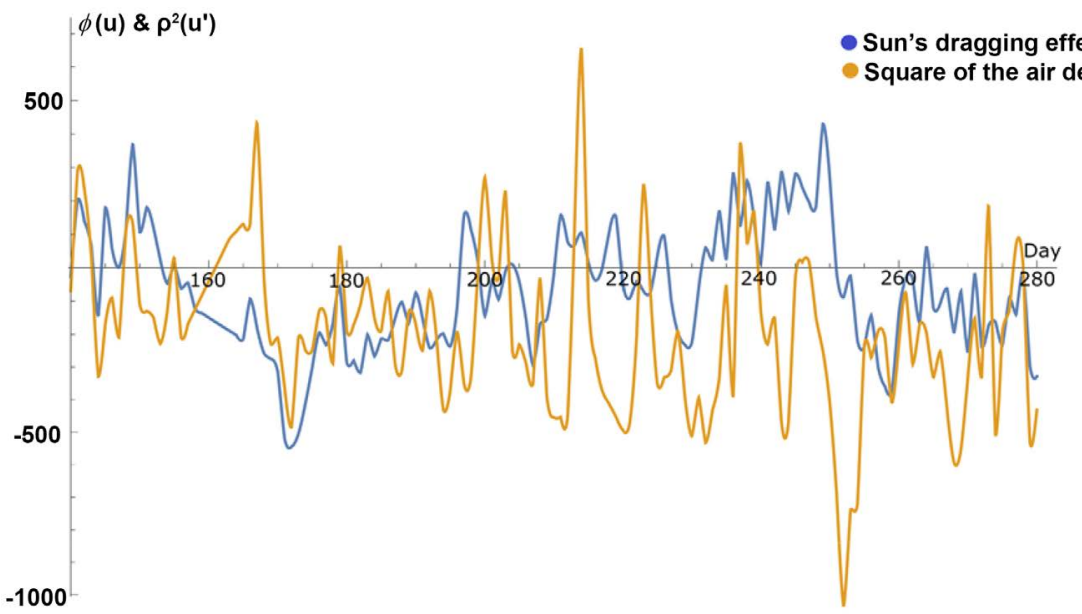

(b)

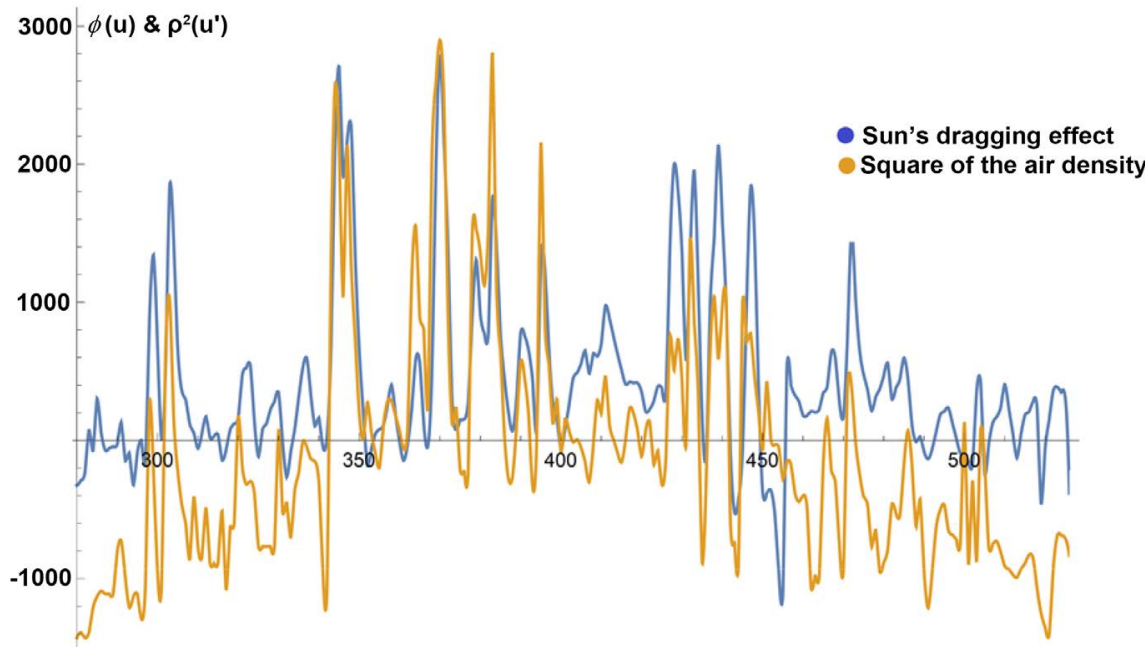

(c)

Figure 3. (a) Data from December 2016 to May 2017; (b) Hurricane season, as notable in our region, with data from May 2017 to October 2017. During this part you can note the relationship between the two parameters is visible but weaker; (c) Data from October 2017 to June 2018 . 
show the overlapping of deflections produced by the Sun, after removing the variation created by the two-year precession, and the density squared of the air. Notice that there is always a perfect match between the picks of both data points. It is important to clarify that both sets of points come from two separate localities and then the cause of those peaks must be external to both data sets. Another argument in favor of this idea is that outside of the peaks every set follow unlikely patterns.

This coincidence between a gravitational balance fluctuation and the density of the air is pointing to the conclusion that maybe the so called tidal forces on Earth are a direct consequence of the dragging effects created by the rotation of the Sun's torus. If true, the construction of some gravitational stations around the world could become one of the most reliable methods of collecting information about weather forecast conditions.

In Figure 3, relative units were used to make possible the superposition of both data sets. Specifically, the Sun's perturbation was forced to be almost horizontal, the square of the air density was amplified to make a distinction among the peaks, and, those oncoming numbers were offset some how to make possible the desire superposition.

Now it is necessary to point out the limitation of the experimental data collected by the author. The possible correlation between the Sun's dragging forces and the completely weather parameters affected by those forces, for example the tide levels, will require funds far above of the author capacity. The author makes contact with two forecast stations, but they show no interest.

This pattern tempts our curiosity with the question "If the density of the air change in Miami with the pick of the Sun's dragging effects, what is happening in other regions of the world?".

\section{The 2017 Total Eclipse of the Sun}

Pugach [9] reported that an eclipse of the Sun happening in the other side of the planet creates observable variations on his balance. That aim the author to quantify that effect. In August $21^{\text {st }}, 2017$ a total solar eclipse was visible in the United States, which from the point of view of Miami depicted that $4 / 5^{\text {th }}$ of the surface of the Sun was covered. Two sets of data were recorded to understand if occulting the Sun can create variations in its dragging forces. The horizontal dragging angles were taken every 15 minutes the day before the eclipse and the day of the eclipse. The data were collected on both days from 5:30 A.M. to 8:00 P.M. The mode in which the dragging angle was changing with the eclipsing-time brought new information about the nature of the dragging forces.

The points for August 21st, 2017 show that the dragging force was decreasing before the eclipse started to occur at 12:00 EST. The Miami local time, which operates in the Eastern Time Zone, will be used in this paper because it is where the dragging-sensor is located. Between 10:00 A.M. and 12:00 P.M. an apparent secondary maximum is observed, its peak happens at 11:00 A.M. From 12:00 P.M. to 18:00 P.M., the eclipse-time, the dragging angle shows a continuous in- 
crement and then starts decreasing after that.

Something unexpected happens when the eclipse is at its maximum, around 15:00 P.M.; after that the dragging angle does not decrease as anticipated; instead, the opposite is evidenced. It is the slope, however that, kept increasing.

A single idea can explain these experimental results. It is, the secondary peak, the continuum increase slope, and the decreasing of the dragging perturbation only after the eclipse was finished, can be the consequence of a simple reason: the dragging force are produced for a sort of coherent photons that come from a direction no coincident with the visual one and satisfying the angle indicated by the Kerr's Metric [10].

The Moon taken as an opaque disk to the Sun's beam, would produce the so-called Fresnel's Diffraction on the Earth's surface. Because the surface of the Moon is not smooth, the Aragon's Spot will not have the same intensity of the open space; the experiments on this topic indicate different rations in correspondence with roughed of the rim of the Moon's surface. The secondary maxima mentioned before matches well with this description. Figure 4 shows the experimental points for the control-day (the day before the eclipse), the points for the eclipse-day and the superposition of Fresnel's Diffraction for a spatial periodicity of $\lambda=6.1 \mathrm{~km}$. The correspondence between time and distance was calculated by how much distance the Moon travelled in the seven hours of the elapsing eclipse-time. The corpuscular equation from [11] was used here as Equation (4), but now abandoning the dimensionless variable $v$ and returning to normal metrical distances $x$ and $y$,

$$
\begin{aligned}
& I_{I}=I_{0}\left\{\left[\cos \left(\varphi_{0}\right)-\left(\frac{y_{i n i}}{y}\right) \cos \left(\varphi_{1}\right)\right]^{2}+\left[\sin \left(\varphi_{0}\right)-\left(\frac{y_{i n i}}{y}\right) \sin \left(\varphi_{1}\right)\right]^{2}\right\} \\
& \text { where } \varphi_{o}=\frac{2 \pi}{\lambda} x \text { and } \varphi_{1}=\varphi_{i n i}+\frac{2 \pi}{\lambda} \sqrt{\left(x^{2}+y^{2}\right)}
\end{aligned}
$$

Of those equations, $x$ is the Earth-Moon distance, $y$ is any radial distance on the surface of the Earth, $\lambda$ is the signal spatial periodicity, $y_{i n i}$ is the distance outside of the Moon that produce the "refraction" and "reflection" of the coming photons, and $\varphi_{i n i}$ is the necessary initial phase that guarantee the continuity between the shadow and illuminate signals. The values used on Equation (4) were tuning by trial and error, those parameters can be deduced rigorously using the model described in [11]. They were $\mathrm{A}=0.147, \mathrm{y}_{\text {ini }}=3807 \mathrm{~km}, \varphi_{\text {ini }}=-2.58 \mathrm{rad}$, and $\lambda=6.1 \mathrm{~km}$. This is the first time that any scholar reported that aravitational effect is fashioned by the laws of optic, which was just as surprising to the author.

The visually complete Sun eclipse occurred at the 15-hour mark. The maximum dragging point, the Arago's Spot, occur at the 11-hour mark. During those four hours, the Moon travelled 14,720 km. Because the Earth-Moon distance is $384,000 \mathrm{~km}$, the dragging photons come at 2.2 degrees inclined respect to the visual focal point. As was mentioned before, the Kerr's dragging forces are not radial and show lines similar to galactic arms. It is important to mention that the 


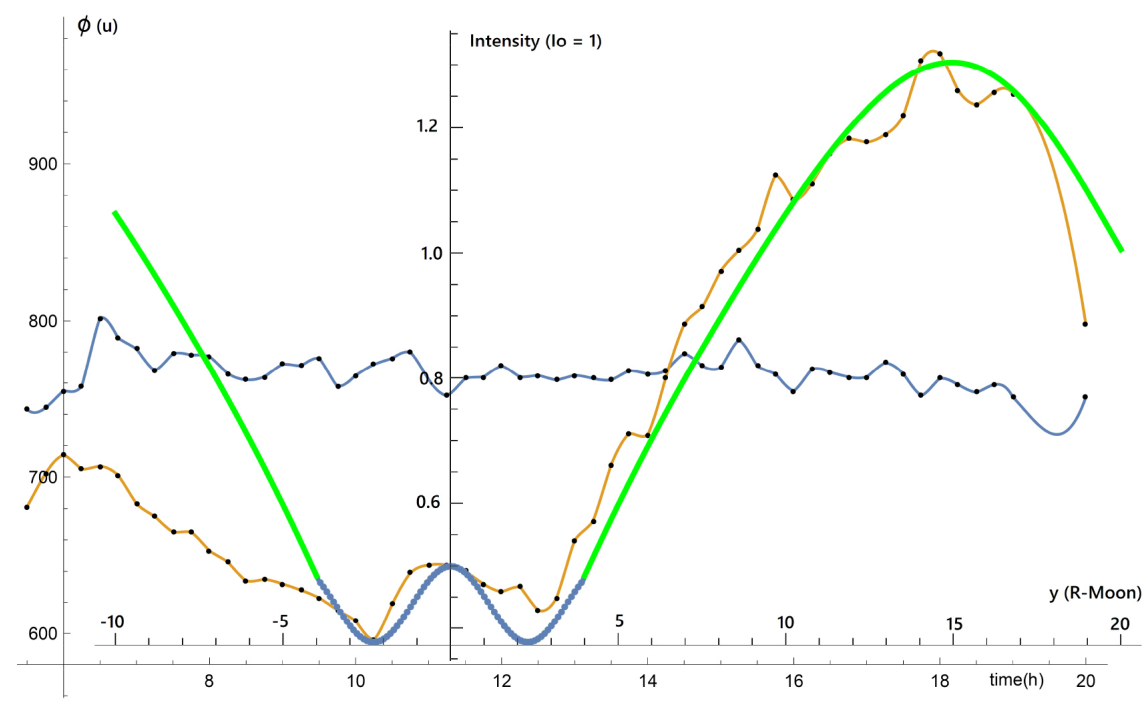

Figure 4. Points with dark-blue connectors for Sun's dragging effect on August $20^{\text {th }}, 2018$, the day before the eclipse. The points of the eclipse-day with connectors in orange. Curves in blue and green for Fresnel's diffraction of an opaque disk of radius equal to the radius of the Moon. The highest Arago's Spot points in blue. On the left side of the figure, experimental and model points have different slope because the rounded of the Earth surface.

Equation (4), describe the interaction between two orthogonal fields, one transversal and the other longitudinal to the direction of the signal of propagation. This idea is working fine for photons, but it is necessary to investigate if it applies to gravitons.

\section{Conclusions}

It looks as even Cavendish struggled with the same main problem that appears in the determination of Big G. He claimed that he could not be more precise that four significant figures on the determination of $G$. Perhaps he concluded that some hard to see systematic error was present in the experiment and stopped doing measurements. More than two centuries later, scholars come to the same conclusion after trying to control the apparatus's error far beyond the third decimal place. A fundamental variation of Cavendish's balance allows the author to make a lineal control of the hidden perturbation. The variation used aimed to change the supporting string numbers. To illustrate, the classical balance used one string, and thus the author used two strings. As a result, manipulating length and the separation of the strings were proved useful to observe microscopic lineal variations on the balance's angles.

Those angle variations showed patterns of at least four periodical movements. The most important of them, discovered by the author, was a Sun's torus flip with a periodicity close to decennia of years and a torus's precession with a period that double the Earth's annual rotation. Those numbers mean that the Earth is in resonance with the Sun's torus frequencies. Due to the torus's flip force, Sun's magnetic field flips its polarity. An inversion of the polarity of the mag- 
netic parameters on one sunspot was observed by an independent Sun-weather station. This coincidence increases the expectation of the model used by the author. If in the next four months the green curve in Figure 2 continues its decreasing tendency, and more reverse sunspots appear, it will provide conclusive evidence in favor of the author's hypothesis.

This paper introduces a new method on the determination of the universal gravitational constant and the dragging parameter. The not so precise equipment settings only allowed the author to make a rough estimation of those values. But more importantly than that there is the experimental verification that the idea works, and the possibility that a more intense accumulation of data could increase appreciably the precision of the gravitational parameters.

An unexpected bonus comes from the observations made by the author, and a clear connection appears between the peaks of the Sun's dragging forces (a direct consequence of the Sun's torus nutation) and the density of air squared. This connection could be a strong tool for weather forecasting. The implementation of some double-string dragging stations could be useful to identify and understand the source of other variations not produced directly by the Sun but hid by its signal.

A total lunar eclipse on regions close to the author's gravitational station produces variations of the dragging forces. The mathematical characterization of those variations pointed in the direction of optical nature of the dragging effects. It looks as if the dragging forces are carried out by radio photons of a large spatial periodicity. In the case that this result becomes confirmed by other scholars, it will be the first announcement of a new common ground for gravity and optics.

\section{Acknowledgements}

My sincerest gratitude goes to Natalie Garcia for her efforts aided the careful editing and correction in the style followed in this paper.

\section{Conflicts of Interest}

The author declares no conflicts of interest regarding the publication of this paper.

\section{References}

[1] Schlamminger, S., Gundlach, J.H. and Newman, R.D. (2015) Recent Measurements of the Gravitational Constant as a Function of Time. Physical Review D, 91, Article ID: 121101. https://doi.org/10.1103/PhysRevD.91.121101

[2] Stirling, J. (2017) A Vector Field Approach to Calculating Gravitational Forces. New Journal of Physics, 19, Article ID: 073032. https://doi.org/10.1088/1367-2630/aa7c80

[3] Parra, J.L. (2017) The Implications of the Sun's Dragging Effect on Gravitational Experiments. International Journal of Astronomy and Astrophysics, 7, 174-184. https://doi.org/10.4236/ijaa.2017.73014 
[4] Schwabe, M. (1849) Solar Sunspot Cycle of 11 Years. Astronomische Nachrichten, $21,234$.

[5] Spaceweather.com. (2018). http://www.spaceweather.com

[6] Pitkin, M. (2015) Comment on “Measurements of Newton's Gravitational Constant and the Length of Day" by Anderson J. D. et al.. EPL, 111, Article No. 30002. https://doi.org/10.1209/0295-5075/111/30002

[7] Desai, S. (2016) Frequentist Model Comparison Tests of Sinusoidal Variations in Measurements of Newton's Gravitational Constant. EPL, 115, Article No. 20006. https://doi.org/10.1209/0295-5075/115/20006

[8] MesoWest, Utah University (2018). http://mesowest.utah.edu/cgi-bin/droman/mesomap.cgi?state=FL\&rawsflag=3

[9] Pugach, A. (2015) Diurnal Variations and Spikes by the Torsind Registered and Their Impact on the Accuracy of G Measurement. International Journal of Astronomy and Astrophysics, 5, 28-37. https://doi.org/10.4236/ijaa.2015.51005

[10] Kerr, R.P. (1963) Gravitational Field of a Spinning Mass as an Example of Algebraically Special Metrics. Physical Review Letters, 11, 237-238. https://doi.org/10.1103/PhysRevLett.11.237

[11] Parra, J.L. (2018) Corpuscular Point of View to Explain Light's Properties. Optics and Photonics Journal, 8, 135-145. https://doi.org/10.4236/opj.2018.85013 